\title{
Intra-operative trans-esophageal echocardiography in heart valve disease
}

\author{
Aayush Poddar $^{1}$ (D) Hyun Suk Yang ${ }^{2} \cdot$ Chandrasekar Padmanabhan $^{1} \cdot$ Joseph Maalouf $^{3}$. \\ Krishnaswamy Chandrasekaran ${ }^{3}$
}

Received: 29 October 2019 / Revised: 11 November 2019 / Accepted: 18 November 2019/Published online: 9 January 2020

(C) Indian Association of Cardiovascular-Thoracic Surgeons 2020

\begin{abstract}
Peri-operative echocardiography is widely used because it provides information that significantly influences clinical/surgical management and improves outcome in patients undergoing cardiac surgery. The role of intra-operative trans-esophageal echocardiography (TEE) in valvular heart disease cannot be emphasized enough. Increasing use of newer surgical techniques-valve repairs and minimal invasive cardiac surgery also warrants intra-operative TEE. It gives us better insight into the anatomy and physiology of the valvular lesion by digital imaging. This manuscript provides an illustrative case based overview of intra operative TEE (IOTEE) in heart valve surgery.
\end{abstract}

Keywords Trans-esophageal echocardiography $\cdot$ Heart valve disease $\cdot$ Intra-operative $\cdot$ Valve repairs

\section{Introduction}

TEE provides high resolution 2-dimensional (2D), 3dimensional (3D), colour flow and spectral Doppler images of the heart which has enhanced the surgical approach to heart valve disease. It has become an indispensable tool in modern cardiac surgery $[1,2]$. This manuscript provides an illustrative case based overview of intra-operative TEE (IOTEE) in heart valve surgery.

\section{General principles}

Planning for surgical intervention includes:

1. Careful thorough pre-operative evaluation of the patient and the details of their investigations. Some may have had

Krishnaswamy Chandrasekaran

KChandra@mayo.edu

1 Department of Cardiothoracic Surgery, G. Kuppuswamy Naidu Memorial Hospital, Coimbatore, India

2 Department of Cardiovascular Medicine, Konkuk University Medical Center, Seoul, South Korea

3 Department of Cardiovascular Medicine, Mayo Clinic, 200 First St. SW, Rochester, MN 55905, USA
TEE as part of their evaluation but in many a good transthoracic echocardiographic (TTE) data are available for preview.

2. Reviewing the pre-operative imaging studies is essential to tailor the exam to answer the question on hand and to best manage time, since time is limited.

3. Familiarity with common devices and surgical procedures enables to anticipate and recognize procedure-related complications.

Although, pre-bypass IOTEE is goal oriented, it should be detailed enough not to miss the findings that have the potential to alter the surgical procedure $[2,3]$. Surgical procedure was altered by the pre-bypass IOTEE in $11 \%$ of cases. Similarly, post-bypass IOTEE is also important as it prompted an additional pump run to improve the outcome in $4 \%$ of cases $[2,3]$.

Essential pre-bypass IOTEE information for any cardiac surgery should include the following as they impact the planned surgical procedure and the outcome:

1. Left ventricular (LV) size and function; regional wall motion abnormality (RWMA)

2. Right ventricular (RV) size and function

3. Screen for intra-cardiac masses especially left atrial appendage (LAA) in patients with dilated left atrium (LA) and atrial fibrillation 
4. Valve pathology-stenosis, regurgitation; these lesions should be assessed at near physiologic loading conditions as anaesthesia may change the loading conditions undermining the severity of these lesions.

5. Screen for significant intra-cardiac or intra-pulmonary shunts

6. Aortic cannulation site, arch of aorta and descending thoracic aorta for complex debris and calcification

Essential post-bypass IOTEE information following any cardiac surgery should include the following:

1. Intra-cardiac air and the magnitude of bubbles and the location

2. $L V$ and $R V$ relative size and qualitative function

3. New RWMA, the extent and magnitude

4. Qualitative assessment of the operative result, if valve repairs, quickly look at the integrity and reassess with optimum loading conditions

5. Quick survey of cannulation site after de-cannulation

6. Quick survey of pleural spaces especially left side for collection of blood

\section{Aortic valve surgery}

\section{Aortic stenosis}

The primary role of IOTEE is to assess the aortic root for size, aortic valve, and the proximal ascending aorta (AA). The primary goal is not to assess the severity of stenosis, which should have been established by a TTE at the time of evaluation but to identify any other pathology that may alter the plan or need to be addressed at the time of surgery [4]. However, not infrequently, moderate to severe aortic stenosis (AS) is encountered in patients undergoing coronary bypass surgery, especially those with LV dysfunction with low stroke volume. In these patients, assessing the morphology of the valve and the excursion of the leaflets, extent of calcification, and valve area measured by planimetry may help in deciding whether valve replacement is required in addition [5]. The commonest cause of AS in the elderly patients is degenerative calcification of the aortic cusps. Not infrequently, the calcification may extend to the aortic annulus and aorticmitral curtain and involve the anterior mitral leaflet (AML) causing mitral stenosis. Therefore, one of the functions of IOTEE is to help decide whether mitral valve (MV) intervention is needed or not? [4]. Similarly, post stenotic aortic dilatation is another entity where IOTEE by providing the details of aortic geometry will help in the decision-making whether aortoplasty or conduit repair is needed. AS from post inflammatory aetiology such as rheumatic fever, frequently encountered in under-developed countries, is characterized by thickening and retraction of the cusp edges and fusion of the commissures. Calcification and restricted mobility and opening of the bicuspid aortic valve are the most frequent causes of AS in middle-aged adults. Very rarely, noncalcified or very minimal calcification of the raphe is seen with congenital AS in younger adults. In these scenarios, valve repair such as peeling the scar tissue, decalcification to improve the mobility, and coaptation, creating a tricuspid valve (TV) by splitting the conjoined cusp of a bicuspid valve to improve the opening excursion is feasible in experienced centers [6].

\section{Pre-bypass IOTEE information}

1. Aortic root morphology and geometry: Dimensions of the aortic annulus, sinus of Valsalva, sino-tubular junction, and ascending aorta are essential (Fig. 1).

Aortic annulus size measured by TEE correlates well with intra-operative sizing [7]. Assessing the annulus size is helpful, if a homograft is going to be used, to prevent unnecessary time waiting for the valve to thaw. Small annulus less than $2.0 \mathrm{~cm}$ may require root enlargement depending on the body surface area of the patient to prevent patient-prosthesis mismatch. Annulo-aortic ectasia, asymmetry of the aortic root, and ascending aortic dilatation are frequently encountered in AS with bicuspid aortic valve. Careful imaging of the aortic root will disclose dilatation of the sinus of Valsalva, effacement of sinotubular junction (STJ), and dilated proximal ascending aorta [8]. If this is present depending on the size of the annulus, sinus, STJ, AA valve replacement with root replacement, or conduit repair of AA will be determined.

2. Aortic root angulation: Uncoiling of the aorta as a result of atherosclerosis, hypertension, or post stenotic dilatation may at times result in the protrusion of the basal septum into the aortic inlet, narrowing of the left ventricular outflow tract, (LVOT) and may cause dynamic obstruction during systole. In this scenario, septal reduction may be required along with valve replacement.

3. Co-existing dynamic obstruction: Morphology of the functional LVOT between the basal septum and anterior mitral leaflet extending from aortic-mitral curtain is essential to exclude sub-aortic ridge, membrane, co-existing hypertrophic cardiomyopathy (HCM). Systolic anterior motion (SAM) of the anterior mitral leaflet, bright scar on the basal septum from contact lesion, mosaic colour flow turbulence in the LVOT, and characteristic daggershaped spectral envelope of continuous wave (CW) Doppler across the LVOT will allow easy recognition of co-existing HOCM (Fig. 2).

4. Co-existing fixed sub-aortic obstruction: Restricted mobility of the basal anterior mitral leaflet just below the aortic annulus, colour flow turbulence in the LVOT, and 
Fig. 1 Intra-operative TEE image of the aortic root showing the measurement taken at different levels. a aortic annulus; $\mathbf{b}$ sinus; $\mathbf{c}$ sino-tubular junction; $\mathbf{d}$ ascending aorta

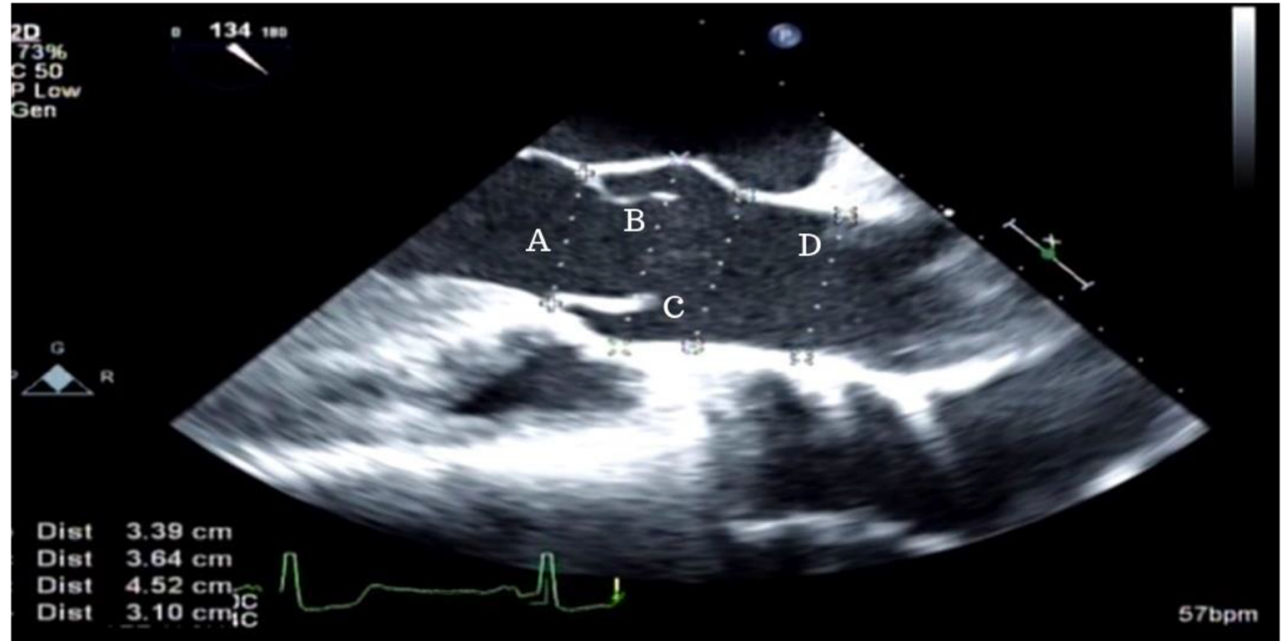

increased flow velocity $>25 \mathrm{mmHg}$ by $\mathrm{CW}$ Doppler should alert to the presence of sub-aortic membrane or ridge resulting in fixed stenosis. Although Doppler profile will be similar to fixed AS, at times, the co-existing dynamic obstruction may make the diagnosis difficult by overshadowing the Doppler profile (Fig. 3).

5. Aortic valve repair may be considered in young patients especially women of childbearing age, competitive athletes if the morphology is suitable - bicuspid valve with well-defined three sinuses, no or minimal calcification, or dense scar tissue amenable for peeling. The measurements essential for valve repair are shown in (Fig. 4).

\section{Post-bypass IOTEE information}

1. Aortic annulus-aortic prosthesis: Seating of the prosthesis and its stability, sewing ring integrity with annulus, with aorto-mitral curtain, with membranous septum anteriorly, and with atrio-ventricular septum posteriorly near the crux should be done meticulously. This will permit recognition of para-valvular regurgitation, tiny communication which may later become a fistula between LVOT and LA, LVOT to right atrium (RA) (acquired Gerbode defect). Uneven thickening and uneven brightness of the aortic root should be evaluated carefully to exclude root hematoma.
Fig. 2 Intra-operative TEE images of a hypertrophic cardiomyopathy patient: a Extensive basal septal, thickening; b Turbulence visualized in the LVOT; $\mathbf{c}$ Elongated anterior mitral leaflet; d Dagger-shaped spectral envelope of continuous wave Doppler

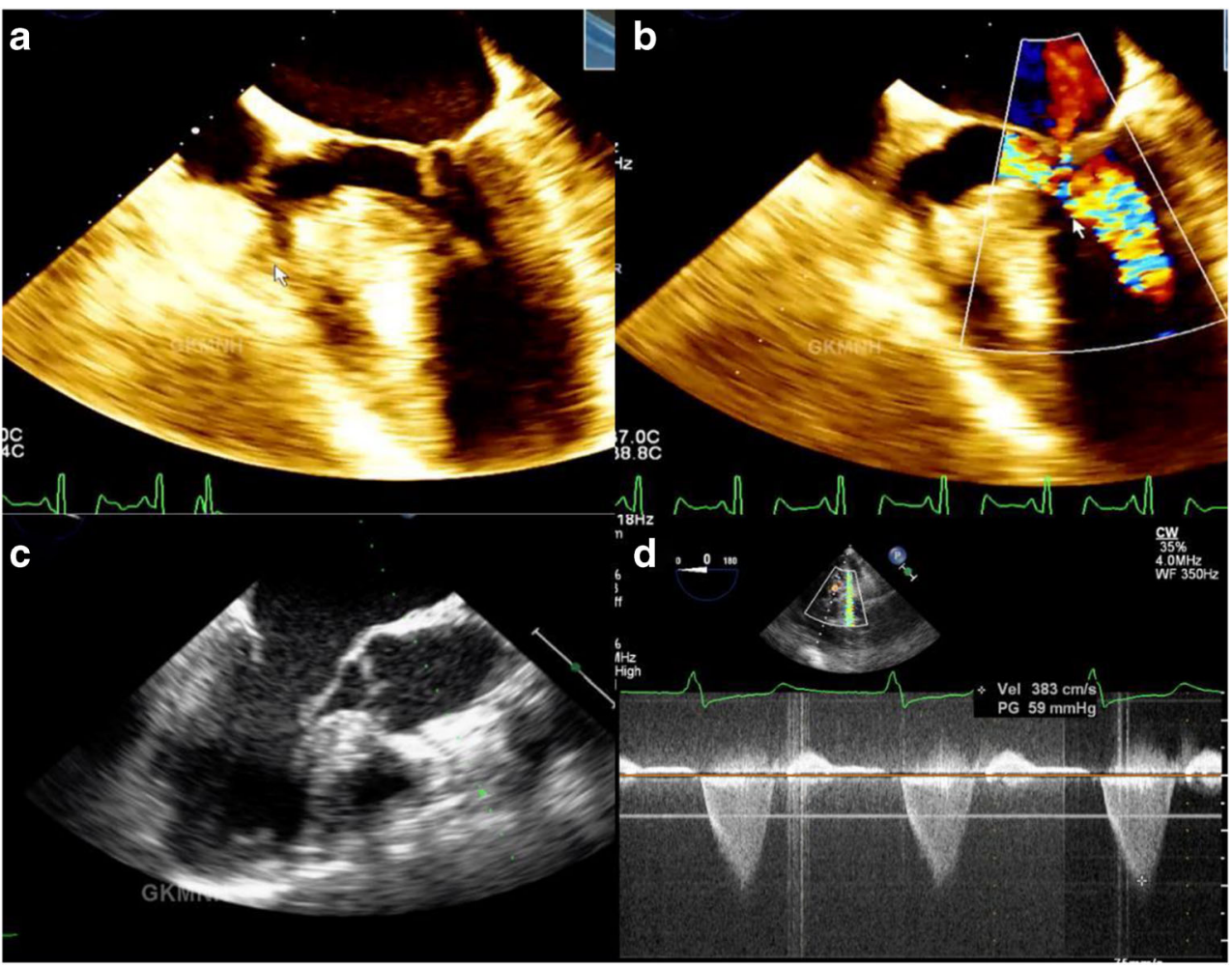


Fig. 3 Intra-operative TEE image demonstrating both components of obstruction: fixed and dynamic

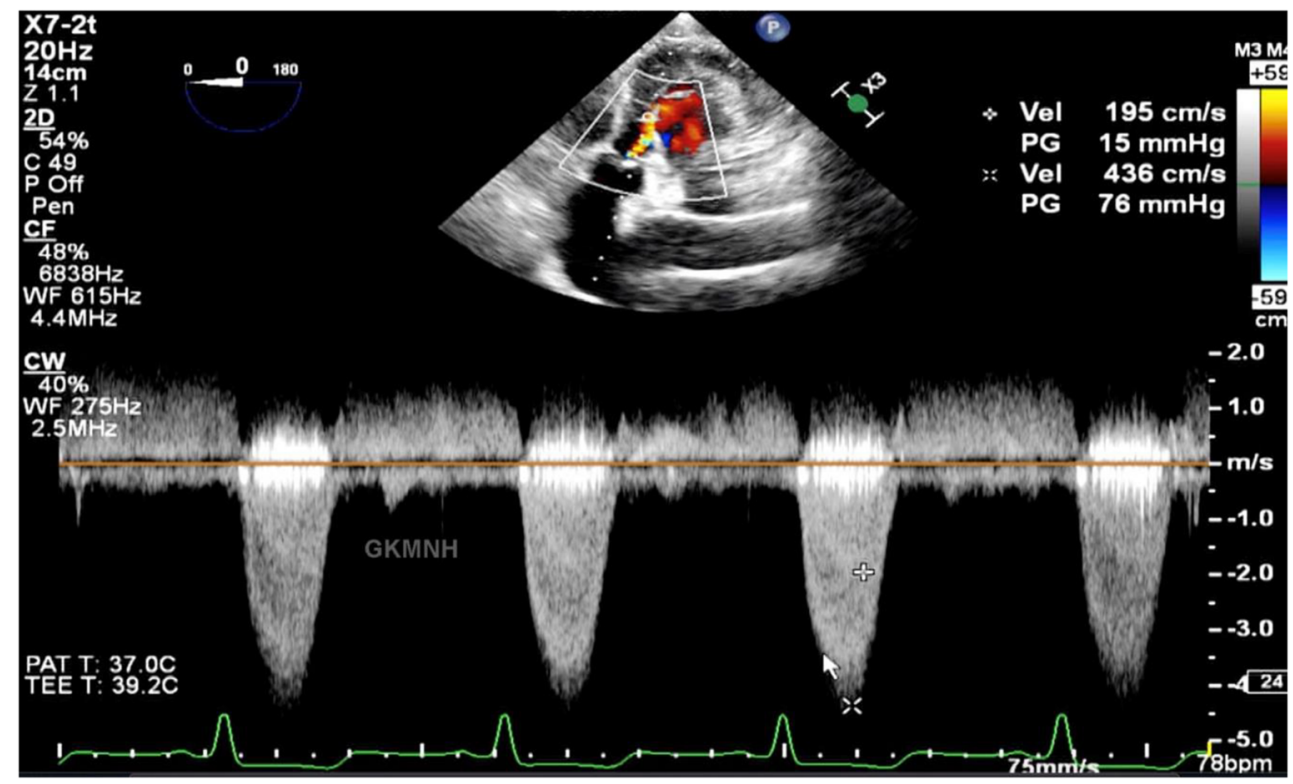

2. Aortic prosthesis: Evaluation which requires imaging the prosthetic disc opening and closure in the case of mechanical prosthesis and leaflet opening and closure in the case of tissue prosthesis both in the longitudinal and in the cross-sectional imaging planes with and without colour flow is essential. Longitudinal imaging of the prosthesis and LVOT can be accomplished from trans-gastric biplane imaging view. This view will also permit obtaining gradients across the prosthesis.

3. Aortic regurgitation (AR): Trivial washout regurgitation is common in mechanical prosthesis and infrequent in tissue prosthesis. If the regurgitation is occurring adjoining the sewing ring, care must be taken to assess the degree and location using multiple conventional and

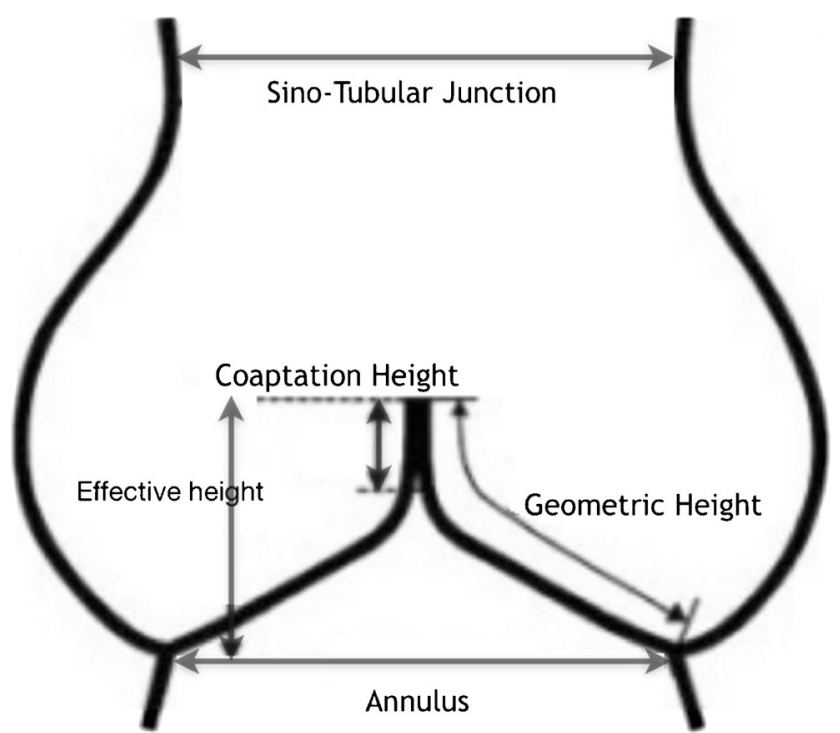

Fig. 4 Schematic diagram showing the aortic root and valve dimensions [9] unconventional imaging planes. More than mild paravalvular regurgitation requires correction by going back on cardiopulmonary bypass (CPB); hence, meticulous identification of the site and degree is crucial. In densely calcified aortic annulus, anterior para-valvular regurgitation may be difficult to pin point; in this situation, transgastric view looking at the LVOT and aortic prosthesis and use of simultaneous bi-plane will help to recognize the site and the severity of regurgitation. Colour flow vena contract width of $<3 \mathrm{~mm}$ generally indicates mild regurgitation [10]. Care should be taken to make sure that the loading conditions (pre and afterload) are near normal.

4. RV size and function: One of the unanticipated complications of aortic valve replacement (AVR) is compression of the right coronary artery (RCA) ostium and RV ischemia. Examination of the RV carefully during coming off the bypass is essential to rectify this complication especially if the central venous pressure (CVP) is unexpectedly elevated and cardiac index is low immediately post-bypass. RV dilatation, decreased RV function compared with pre bypass exam, and small size of the LV should raise the suspicion for RV ischemia. Imaging the aortic root and RCA ostium is often difficult after the AVR; however, if the RCA ostium can be imaged well with and without colour flow imaging, it excludes the possibility of RCA ischemia as the aetiology for RV dysfunction.

\section{Aortic regurgitation}

Unlike AS, AR results not only from abnormalities of the valves but also from other etiologies such as sub-aortic membrane, root dilatation, annulo-aortic ectasia, ascending aortic 
aneurysm, and aortic dissection. Therefore, the goal of IOTEE is not only to assess the morphology of the aortic valve but also to define other etiologies if they are present. Aortic valve for repair is entertained in young adults, childbearing women, and athletes, also in lesions such as perforation, prolapse, and avulsion of the cusp; hence, meticulous evaluation of the aortic cusps and root is essential. The goal of IOTEE is not intended to assess the severity but to define the mechanism of regurgitation as well as to identify other pathologies that may alter the plan or need to be addressed at the time of surgery. However, in some patients undergoing surgery for other reasons, AR may need to be assessed to make the decision on whether it needs addressing. Briefly, the following criteria can be used to assess the AR severity: (a) vena contracta of the colour flow AR jet in any imaging plane $\geq$ $0.3 \mathrm{~cm}$; (b) LVOT/AR width ratio $>30 \%$; (c) holodiastolic flow reversal in the proximal descending thoracic aorta. All these parameters indicate AR is more than mild and cannot be ignored.

\section{Pre-bypass IOTEE information}

1. Valve morphology: Type II and type III mechanisms of AR are due to primary aortic cusp pathology. Although most of the time TTE provides reliable assessment of the aortic valve morphology, TEE provides better visualization of the subtle abnormalities of the aortic valve cusp morphology and mobility which are responsible for type II and type III mechanisms of AR [11] (Figs. 5 and 6).

In majority of the adults, the AR is part and parcel of AS and AVR with mechanical or tissue valve will be required. However, valve repair is an option in younger patients with congenitally abnormal valve such as bicuspid aortic valve (BAV), post inflammatory (rheumatic, connective tissue disorder), or infective endocarditis (IE) aetiology. BAV with well-defined three sinuses, adequate cusp height, and no or minimal calcification can be repaired by creating the third cusp with good success at experienced centers. Repair techniques such as peeling off the scar tissue, leaflet lengthening to improve coaptation height, and primary closure or pericardial patch closure of the fenestration all require meticulous imaging of the cusps.

2. Aortic root geometry: Type I AR is primarily due to aortic root abnormality. Aortic root geometry, STJ, and ascending aorta abnormality interfere with aortic cusp mobility and coaptation resulting in AR. This permits planning for valve sparing aortic root replacement or aortoplasty. The essential dimensions of the aortic cusp, aortic root, STJ and ascending aorta are well described in the literature (Fig.1).
3. Mitral valve morphology and function: Severe AR invariably affects the anterior mitral leaflet and impairs the opening during diastole. If the AR is due to endocarditis, the infection may extend into the aortic root and aorticmitral junction and involve the AML [12]. Imaging the mitral valve and aortic-mitral inter valvular fibrosa is crucial if the indication for AVR is AR from endocarditis.

4. Intra-cardiac fistulous communication: AR from infective endocarditis aetiology requires meticulous imaging of the aortic annulus for abscess, abscess burrowing into various adjoining structures such as atrio-ventricular septum, inter-atrial septum, aortic root to LVOT, aortic root to LA, and RA fistulae. These abnormalities may alter the plan of surgery as well as the use of homograft (Fig. 7).

\section{Post-bypass IOTEE information}

1. Integrity of the valve repair: Cusp excursion, prolapse, location and magnitude of residual regurgitation, and Doppler flow gradients. Note it is better to reassess these once the arterial pressure and volume status are near normal.

2. Integrity of the Prosthetic valve: Disc/leaflet opening and closing, washout jets of regurgitation in mechanical prosthesis; leaflet mobility during systole and diastole, site of regurgitation if there is any. The regurgitations are invariably trivial to mild in normal mechanical and tissue prosthesis. Care should be taken to image the aortic annulus and sewing ring unit in cross section to identify paravalvular regurgitation and location (Fig. 8). Any paravalvular regurgitation requires an attempt at quantitation. The abovementioned criteria are applicable for the assessment of severity.

3. In case of aortic root replacement, assessing the integrity of the anastomotic site is essential. Inappropriate narrowing resulting in increased gradient or more than trivial leak at the anastomotic site should be recognized to address immediately. If coronaries are re-implanted in root repair, assess the LV and RV size and for new RWMA.

4. Mitral valve integrity may be compromised in aortic root repair; therefore, it is important to assess the mitral valve motion and mitral regurgitation.

\section{Mitral valve surgery}

In the current era, TEE is essential for mitral valve repair for both stenosis and regurgitation [13, 14]. TEE played a major role in the early days of mitral valve repair and the recent advances in the TEE technology such as $3 \mathrm{D}$ have 
Fig. 5 TEE image showing Right coronary cusp prolapse with a severe AR

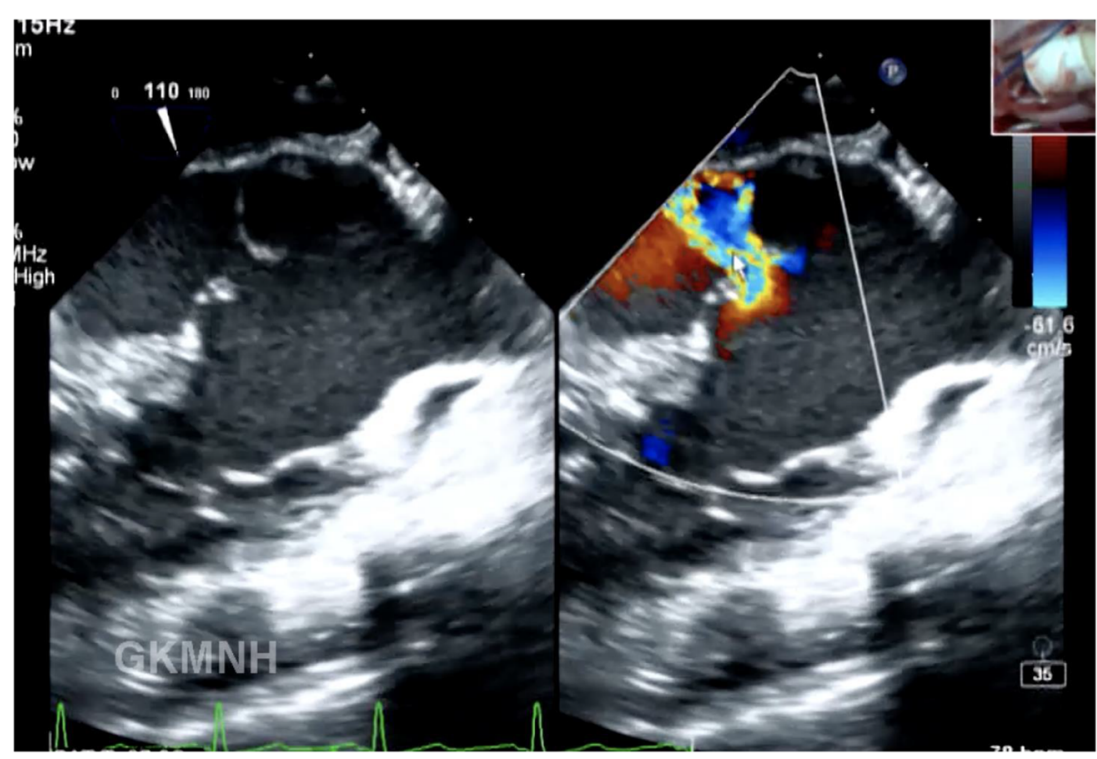

enhanced its value in mitral surgery. TEE provides excellent view of the mitral valve to assess the morphology and this is further amplified by three-dimensional capabilities [13] (Figs. 9 and 10).

3D en-face imaging with its surgical orientation allows surgeon to assess the changes in morphology as well as in colour flow and Doppler imaging the haemodynamic consequences of the pathology in real time during systole and diastole.

\section{Mitral stenosis}

Mitral stenosis (MS) due to rheumatic or post inflammatory aetiology or congenital aetiology repair is feasible. Surgical procedures such as commissurotomy, peeling scar tissue, decalcification of the leaflets, splitting and releasing the chordae etc.; to improve the mobility of the leaflets requires meticulous assessment of the valve morphology $[15,16]$ (Figs. 11 and 12).

\section{Pre-bypass IOTEE information}

1. Establish the aetiology of the stenosis: Is it due to post inflammatory or degenerative pathology? Image the MV in cross-section as well as multiple views. Look at the commissures, chordae, chordal thickness, fusion and retraction, the extent of leaflet excursion, plane of leaflet apposition and plane of closure, papillary muscles, extent of tenting, and the annulus. In degenerative calcific MS, the extensive calcification of the mitral annulus is not uncommon. This may pose a problem for valve replacement. In congenital mitral stenosis, in addition to assessing the valve morphology, it is important to exclude supra-mitral ring, sub-aortic membrane, and coarctation of the aorta.

2. Assess the haemodynamics: Is there associated regurgitation? Identify the mechanism of associated regurgitation, restricted leaflet excursion and mobility due to scar tissue, or calcification, or matting and retraction of chordae? Improving the leaflet mobility and excursion by peeling the calcification/scar tissue and releasing the chordae from matting may decrease the regurgitation by increasing the leaflet coaptation.

3. Assess the LV geometry and the papillary muscle orientation: LV geometry, size, and function; location and function of the papillary muscles are very important if there is co-existing coronary artery disease. MV function, opening as well as closure, can be affected by the changes in LV size, geometry, and displacement and distortion of the papillary muscles. Similarly, RWMA involving the basal inferior and infero-lateral segments will affect the posterior leaflet movement as the tertiary chordae anchor the base of the posterior leaflet to the posterior and posterolateral LV.

4. Aortic root and LVOT geometry: It is important to evaluate the LVOT and aortic root. If the aortic root is uncoiled, the basal septum may protrude into the LVOT and may narrow the LVOT. This information is important in cases of MV replacement to choose a valve with low profile so that it does not obstruct LVOT.

5. Assess the left atrial and LAA: Left atrial enlargement and dilatation of LAA are common in MS. Similarly, atrial fibrillation is frequent; hence, look for thrombi.

6. RV function and tricuspid regurgitation: Long-standing MS will result in pulmonary hypertension with secondary effect on RV function as well as on tricuspid annulus and valve function. Therefore, pre-bypass assessment of RV 
Fig. 6 Phenotypes of the proximal aorta associated with the classification of AR mechanisms [9]

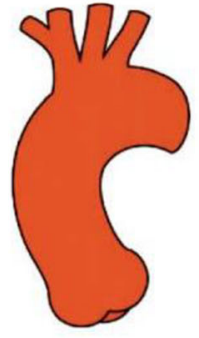

Normal aorta

Type 1

Normal cusp movements related to aortic root or ascending aorta dilation with central jet
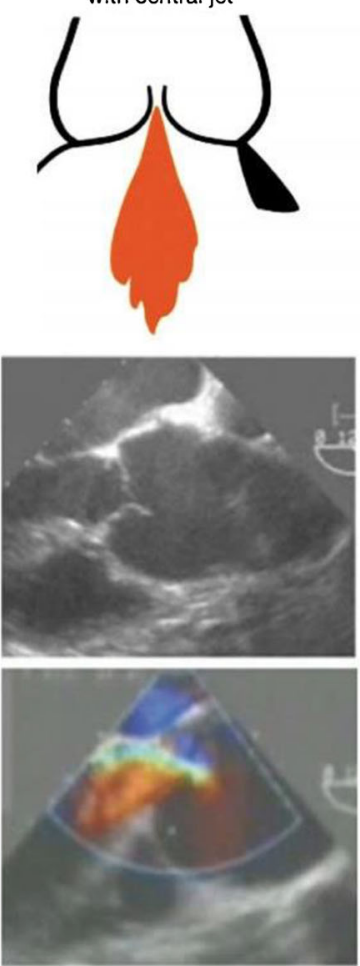

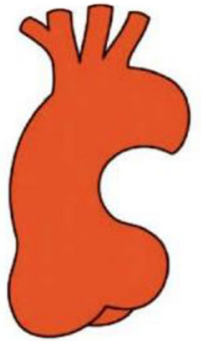

Aortic root dilation

\section{Mechanisms of Al Classification}

Type II Cusp prolapse with eccentric jet
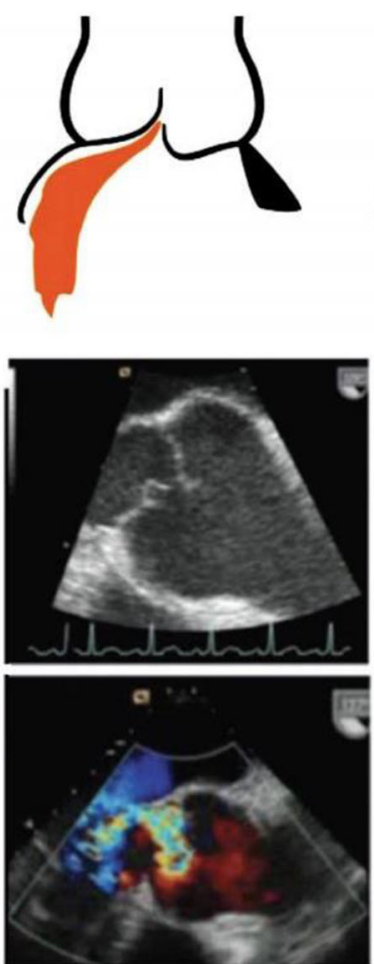

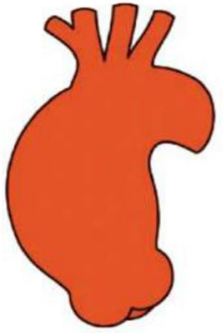

Ascending aorta dilation

Type III Cusp retraction with poor tissue quality or quantity with large central
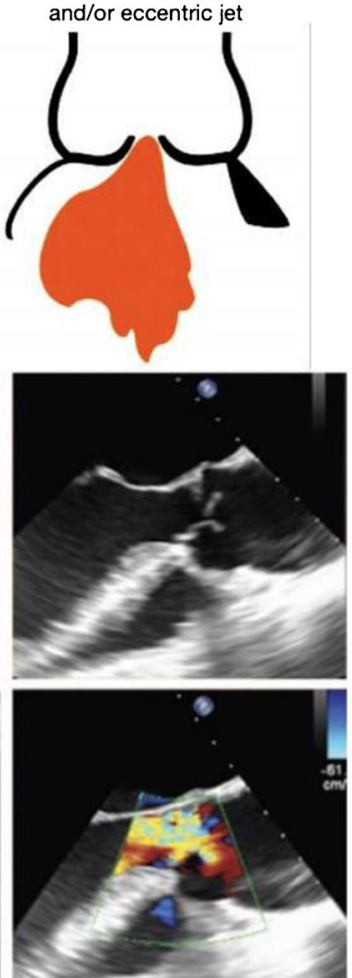

function and status of TV are important to decide whether TV requires any intervention.

\section{Post-bypass IOTEE information}

1. Integrity of the valve repair: Leaflet excursion, location and magnitude of residual stenosis, and Doppler flow gradients. Is there a regurgitation? If so location and severity. Note it is better to reassess these once the arterial pressure and volume status are near normal.

2. Integrity of the prosthetic valve: Disc/leaflet opening and closing, washout jets of regurgitation in mechanical prosthesis; leaflet mobility during systole and diastole, site of regurgitation if there is any. The regurgitations are invariably trivial to mild in normal mechanical and tissue prosthesis. Care should be taken to image the sewing ring unit in cross section to identify para-valvular regurgitation and location. Any para-valvular regurgitation requires an attempt at quantitation.

3. Prosthetic valve closure and opening mechanism: Opening and closing of the prosthetic disc or leaflet can be interfered by chordae from the retained posterior leaflet especially if the chordae are unusually long or redundant.

4. LVOT obstruction: It is important to image LVOT as well as the flow dynamics in the LVOT in case of mitral valve replacement (MVR) in a small LV with angulated aortic root to recognize LVOT obstruction from the prosthesis. 
Fig. 7 TEE demonstrating an Aortic Root to LVOT fistulous tract in a case of Prosthetic Valve Endocarditis

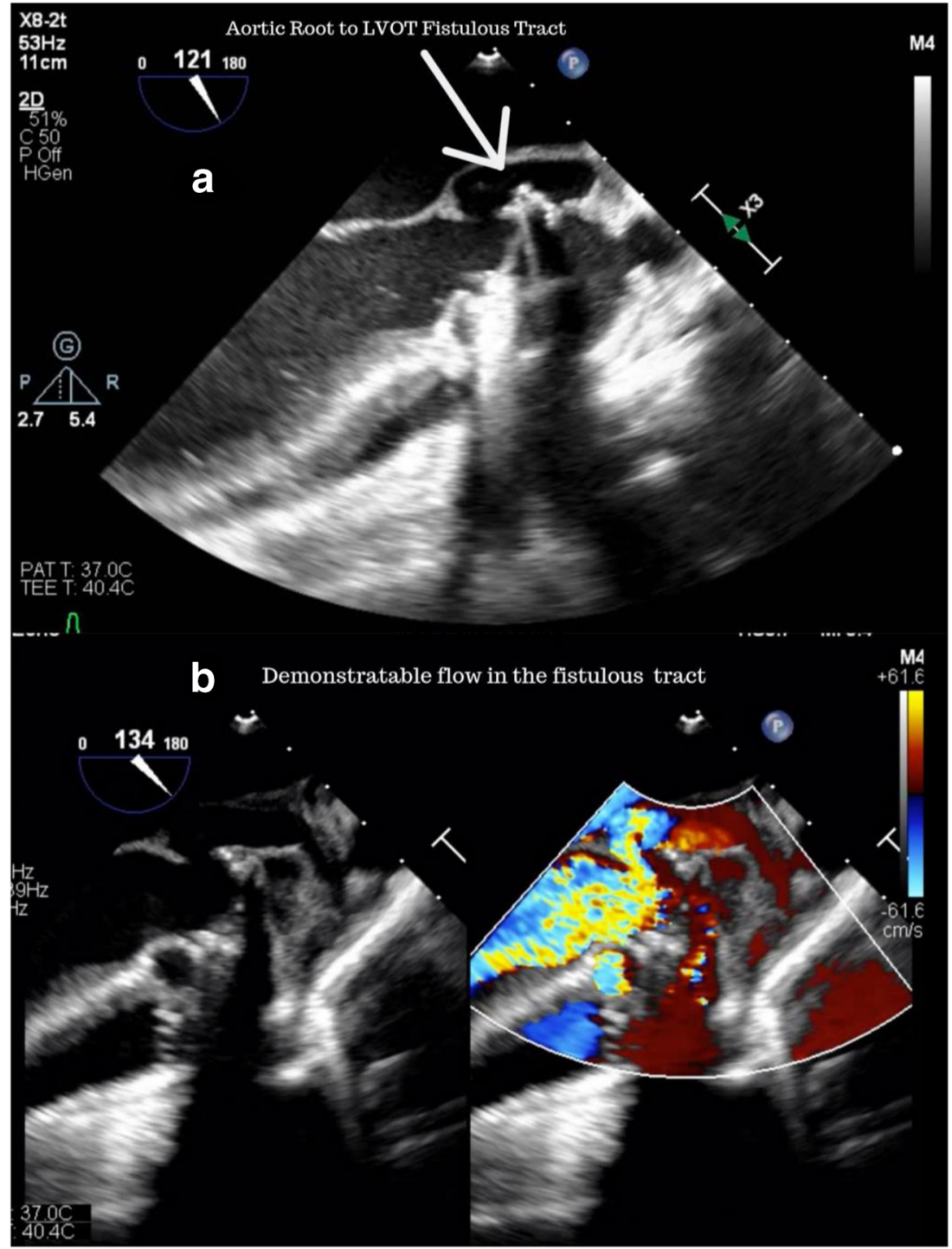

5. LV function and new RWMA: Left circumflex coronary artery can be in advertently ligated during MV replacement resulting in new RWMA affecting the infero-lateral wall.

\section{Mitral regurgitation}

Surgical anatomy of the mitral valve as it applies to valve repair has been well described [17]. TEE is essential to establish the aetiology, mechanism, location, and severity of the regurgitation $[18,19]$. In addition, TEE also recognizes associated pathology such as ventricular dysfunction, pulmonary hypertension, and tricuspid regurgitation (TR) which may have an impact on coming off the CPB. In cases of minimally invasive MV surgery, TEE is also used for guiding the position of cannula used for CPB. 3D en-face imaging of the mitral valve is essential for mitral valve assessment to plan the type of repair. 3D TEE has enhanced the outcomes of mitral repair [10, 20, 21] (Figs. 11 and 12). Functional mitral regurgitation (MR) due to dilated annulus, distorted and retracted papillary muscle, and LV dilatation will require meticulous assessment of leaflet coaptation length, coaptation height, and tenting angle. MR due to endocarditis requires careful assessment for leaflet perforation, flail segment, and adjoining annular integrity for repair.

\section{Pre-bypass IOTEE information}

1. Mitral valve morphology:

a. Mitral - abundance of leaflet tissue and mobility of leaflet tissue (localized vs extensive), perforation

b. Mitral annulus size and angle with aortic root

c. Anterior leaflet length and angle with aortic root

d. Location and extent of annular calcification, TR severity (TTE) 
Fig. 8 a Intra-operative TEE image showing a para-valvular leak after a bio-prosthetic valve replacement. b Image after the paravalvular regurgitation was fixed

Fig. 9 TEE image demonstrating a mitral valve with a flail AML during systole
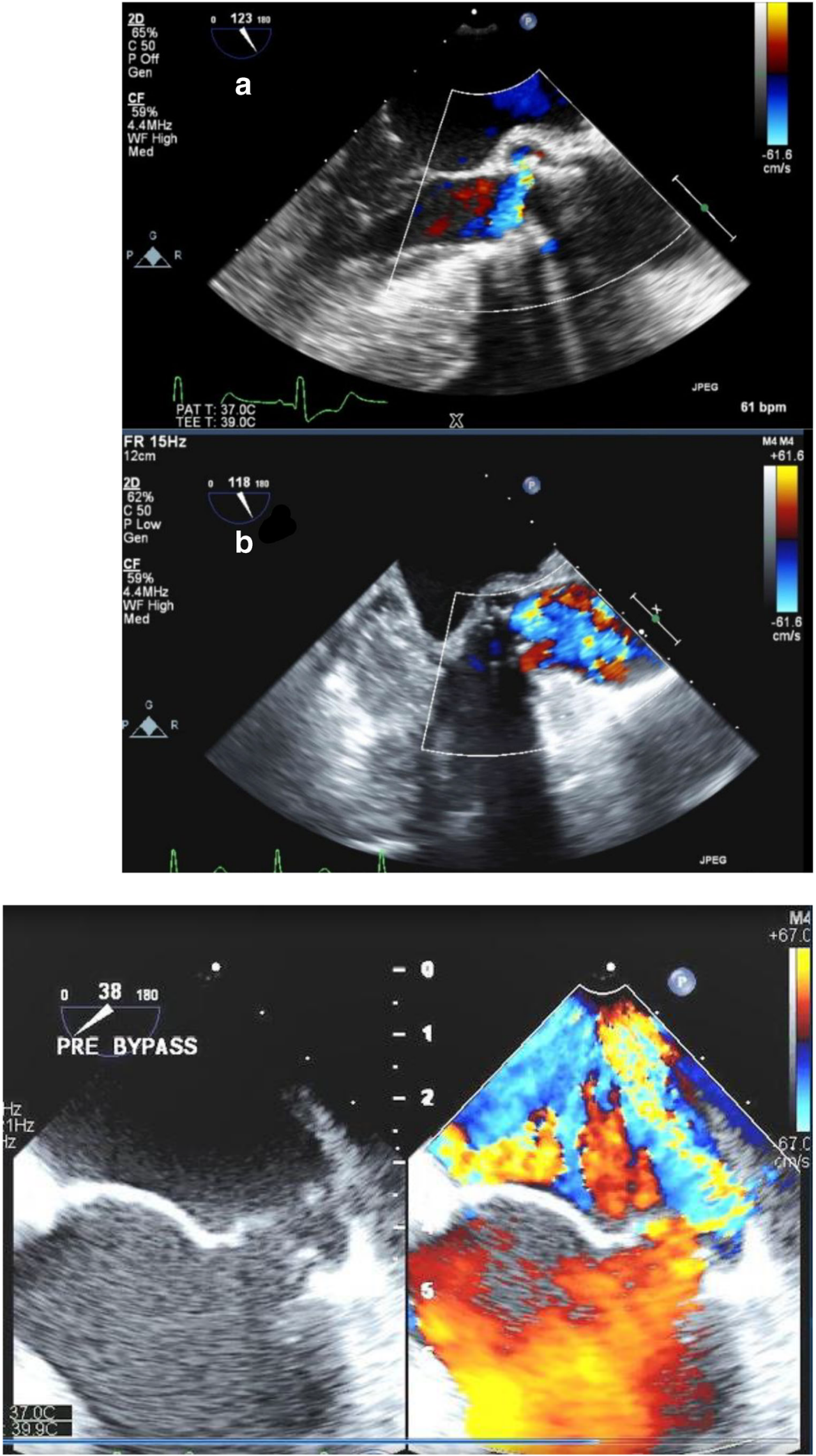


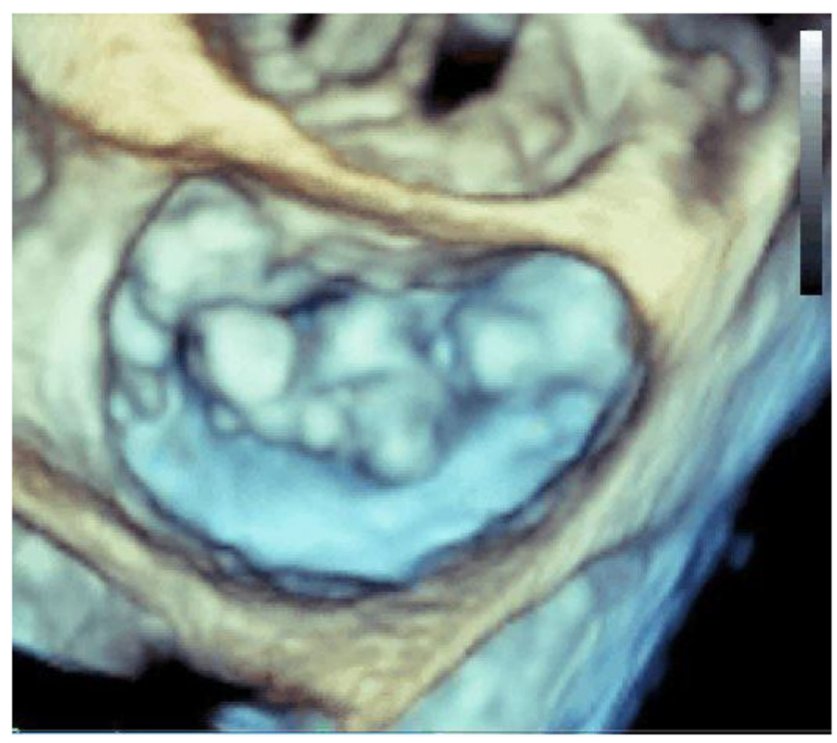

Fig. 10 TEE 3D image showing the en-face surgical image of a mitral valve with flail AML during systole

2. Barlow's disease: Prioritize scallop that is the culprit for major regurgitation

3. Regurgitation: the anatomy of the leak-prolapse, flail, clefts, and commissural integrity

4. Left ventricle: LV geometry, papillary muscle integrity, RWMA, and leaflet tenting

5. Tricuspid regurgitation and RV systolic pressure

\section{Post-bypass IOTEE information}

1. Integrity of the repair: Residual regurgitation and location. Care should be taken to keep the pre- and afterload within normal limits [22].

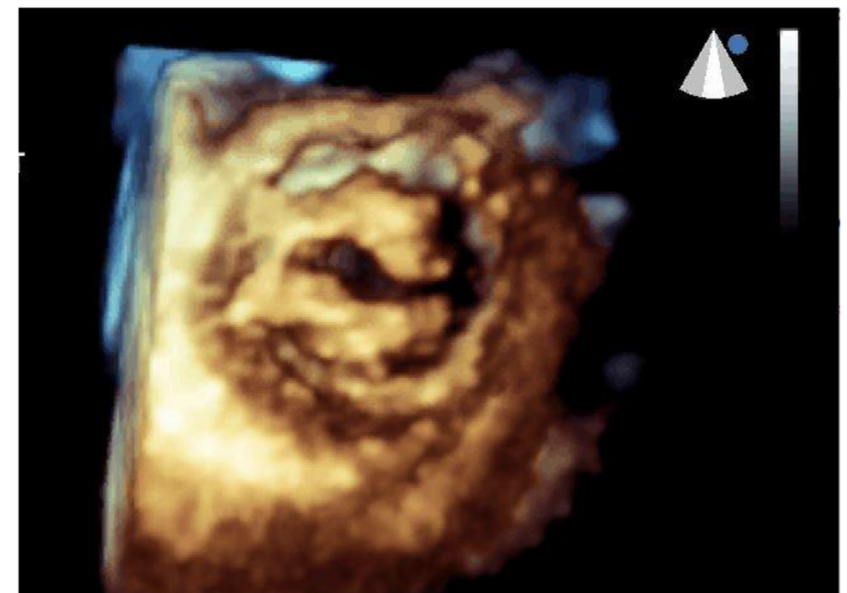

Fig. 12 TEE 3D en-face view of the mitral valve during diastole post commissurotomy

2. LVOT morphology and gradients if there is systolic anterior motion or anterior leaflet is long [23]

3. Aortic valve - not uncommon to distort aortic annulus resulting in regurgitation with mitral annuloplasty repair, usually if complete and rigid ring was used

4. LV geometry and new RWMA

\section{Hypertrophic cardiomyopathy}

IOTEE plays an important role in recognizing the site of anterior mitral leaflet to septal contact and in identifying other causes for mitral regurgitation. It provides anatomical details for planning the extent of myectomy. IOTEE and 3dimensional imaging have shown to be useful in HCM surgery in identifying the septal thickness, location, LVOT geometry, MV, and mitral regurgitation (Fig. 13, 1A-C) [24, 25].
Fig. 11 TEE 3D en-face view of the mitral valve during diastole in a patient with Mitral Stenosis

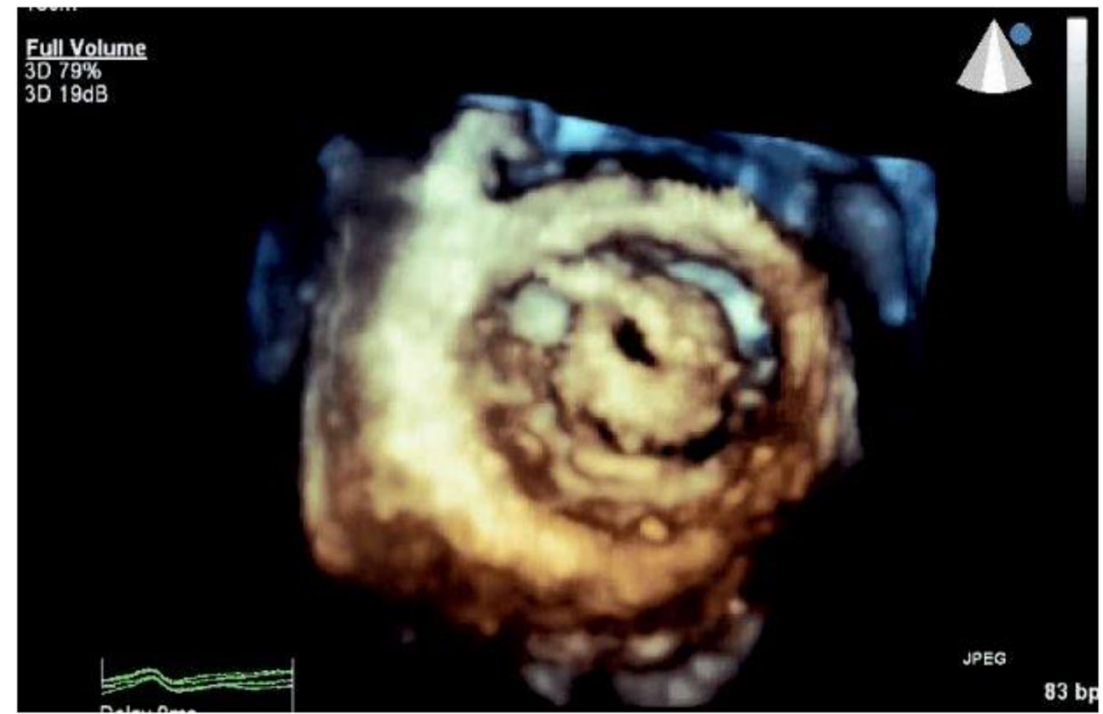



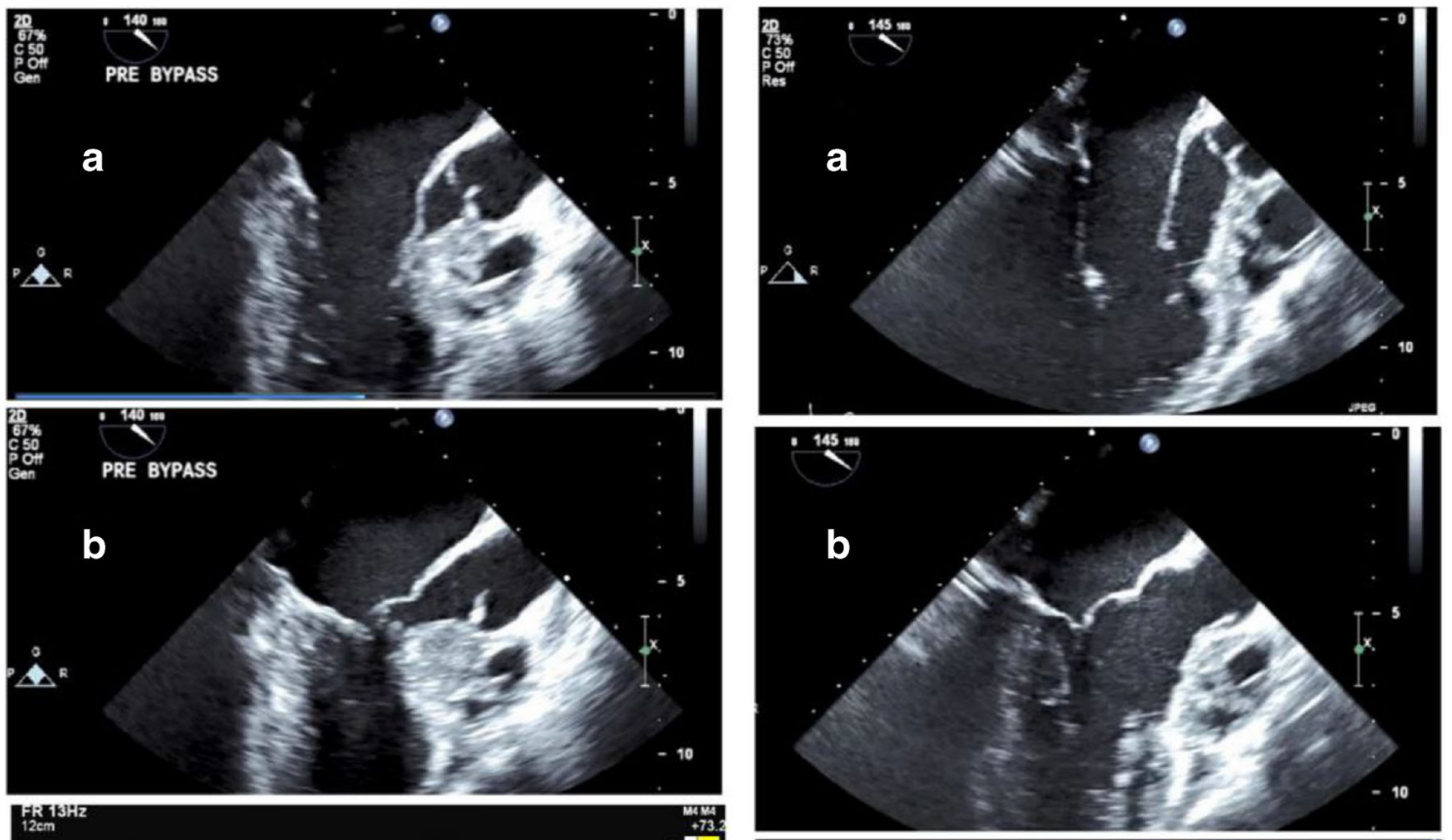

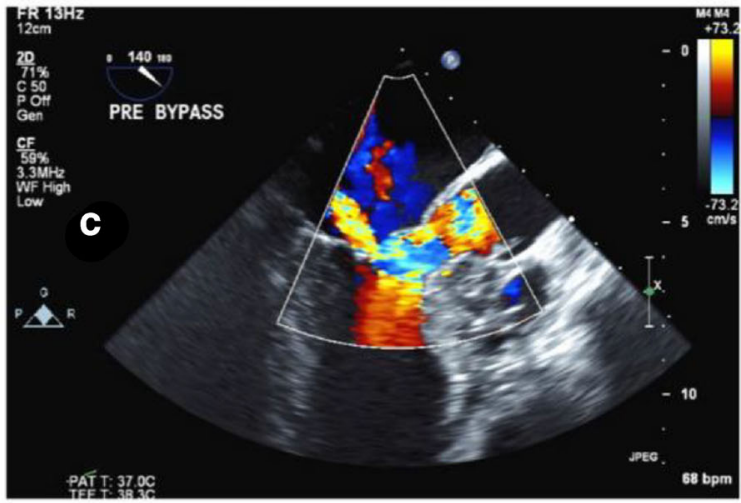

Pre-Operative

Fig. 13 TEE pictures demonstrate asymmetric basal septal hypertrophy before surgery and after surgery. Note the elongated anterior mitral leaflet. Note the narrowed LVOT and systolic anterior motion of the mitral leaflet (1A, B). Colour flow demonstrates turbulence in the LVOT and laterally

Hypertrophic cardiomyopathy is also associated with other lesions and these may be giving rise to the outflow gradients that require meticulous sorting out [26] (Fig. 3). Mitral valve abnormalities are frequent in HCM and may have independent mechanism of mitral regurgitation not related to LVOT obstruction [27]. Similarly, LVOT obstruction may occur from anomalous papillary muscles frequently encountered in these patients [28]. IOTEE also provides important information about residual LVOT obstruction, mitral regurgitation, and complications from myectomy (Fig. 13, 2A-C) [29].

\section{Pre-bypass IOTEE information}

1. Assess the inter-ventricular septum-maximal thickness, thickness at the site of mitral valve contact, distance from aortic annulus, and extent of the septal thickness

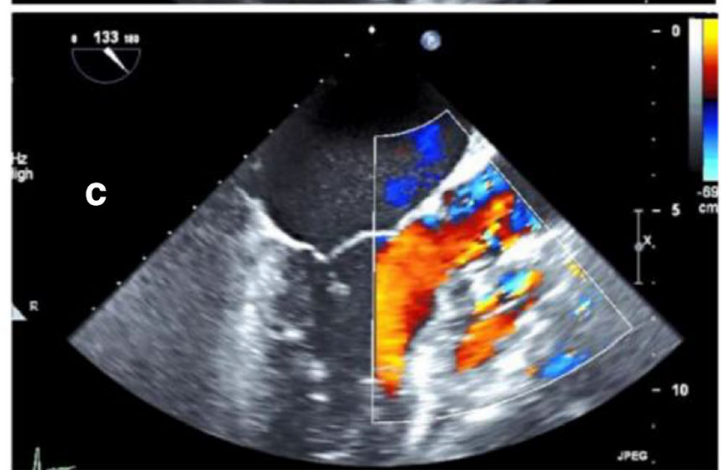

\section{Post-Operative}

directed mitral regurgitation jet (1C). Post-operative pictures demonstrate wide open LVOT and relief of systolic anterior motion of the mitral leaflet and LVOT obstruction (2A, B). Colour flow demonstrates no turbulence in the LVOT $(2 \mathrm{C})$

involving other regions such as anterior wall and inferior septum.

2. Assess the anterior mitral leaflet, chordae tendineae, and accessory muscle bundles.

3. LVOT geometry and flow acceleration by colour flow and by CW Doppler

4. Mid cavity geometry of the LV and intra-cavity flow acceleration

5. Mitral regurgitation and its direction

\section{Post-bypass IOTEE information}

1. LVOT geometry and patency

2. Integrity of the inter-ventricular septum - rarely septal defect can result. 

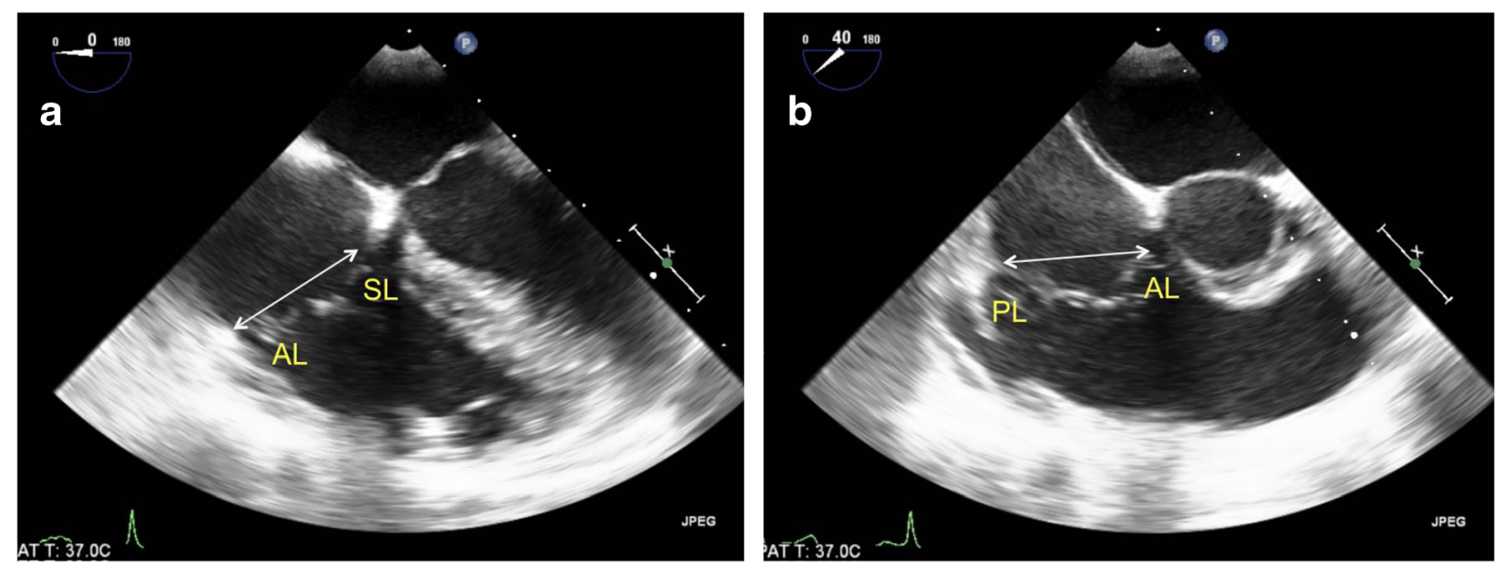

Fig. 14 Tricuspid annulus diameter (arrows) at the end-diastolic phase. a Mid-esophageal four-chamber view. b Mid-esophageal right ventricular inflow view. AL, anterior leaflet; SL, septal leaflet; PL, posterior leaflet of the tricuspid valve

3. Aortic valve integrity-AR

4. Residual intra-cavity and LVOT gradients

5. Mitral valve integrity and degree and direction of regurgitation

\section{Tricuspid valve surgery}

Tricuspid valve assessment by TEE has received renewed attention currently due to increased interest in developing percutaneous techniques for treating tricuspid regurgitation. The common tricuspid pathology is functional tricuspid regurgitation from remodelling of the tricuspid annulus secondary to left heart disease. Similar to mitral regurgitation, the tricuspid regurgitation is also sensitive to pressure and volume changes; therefore, the need for tricuspid repair should be made before the patient arrives in the operating theatre.

\section{Pre-bypass IOTEE information}

1. Assess the TV morphology, leaflet tethering, and mode of coaptation.

2. Tricuspid annulus diameter. Annulus diameter measured from the mid-esophageal apical four-chamber view, a diastolic diameter $\geq 40 \mathrm{~mm}$ or $>21 \mathrm{~mm} / \mathrm{m}^{2}$ indicates severe annular dilatation [30] (Fig. 14a, b).

3. Assess the severity of tricuspid regurgitation - vena contracta $>7 \mathrm{~mm}$, proximal isovelocity surface area (PISA) radius $>0.9 \mathrm{~cm}$ at the baseline Nyquist shift of
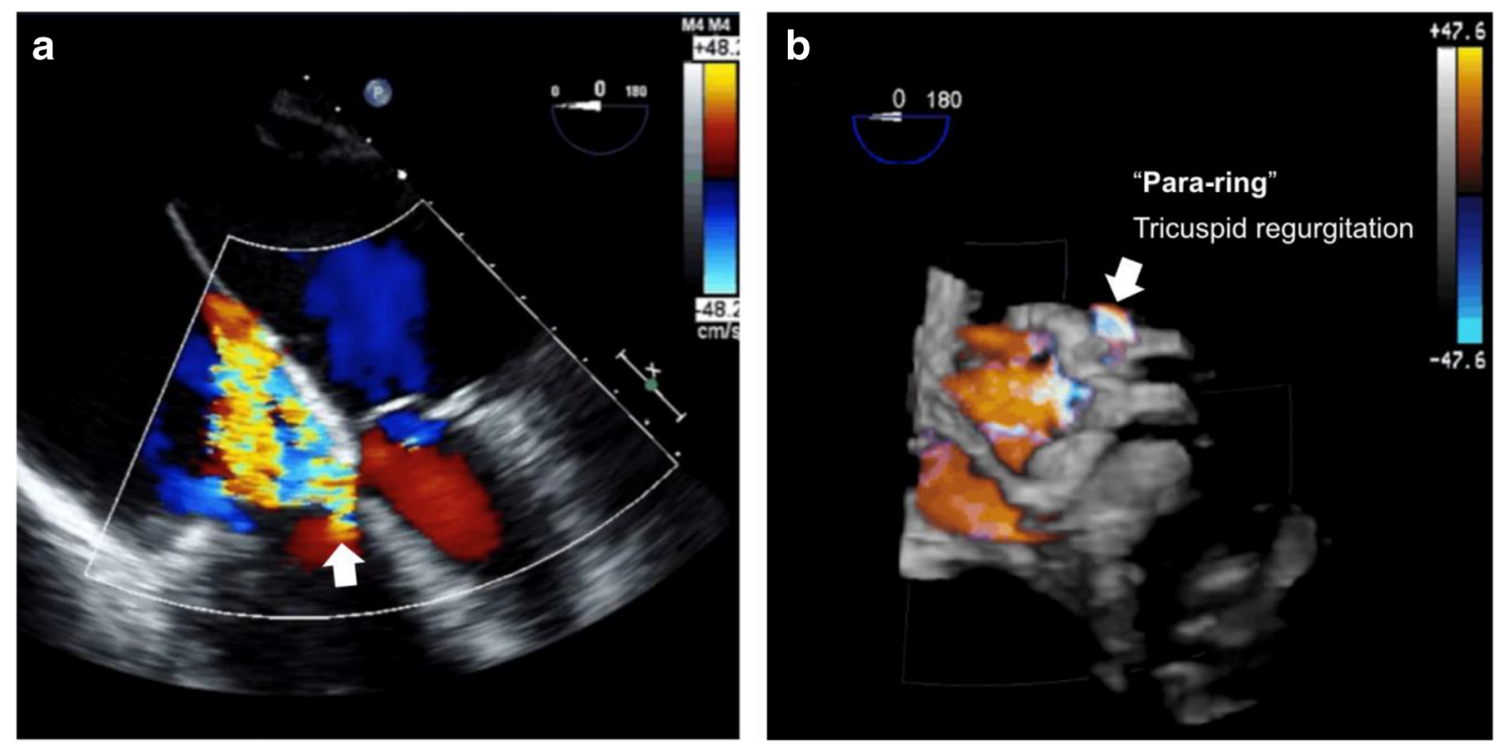

Fig. 15 Post-bypass intra-operative trans-esophageal echocardiography showing para-ring tricuspid, regurgitation (arrow), a leak originating from around the ring, from a mid-esophageal four-chamber view (a) and 3D colour full-volume image (b) 
$28 \mathrm{~cm} / \mathrm{s}$ or regurgitation jet $>50 \%$ of RA or any eccentric wall impinging jet indicates severe TR [31].

4. Assess the RV size and function.

\section{Post-bypass IOTEE information}

1. Assess the TV diastolic gradient to make sure that there is no haemodynamically significant stenosis.

2. Assess the degree of residual regurgitation; make sure the RA pressure is closer to pre-bypass value (Fig. 15).

3. Assess RV function as TR may be secondary to RV dysfunction.

Funding information The authors received no financial support for the research, authorship, and /or publication of this article.

\section{Compliance with ethical standards}

Conflict of interest The authors declare that they have no conflict of interest.

Animal rights This article does not contain any studies with animals performed by any of the authors.

Informed consent Informed consent was obtained from all individual participants included in the study.

Disclaimer The paper reflects the authors' own research and analysis in a truthful and complete manner. All authors have been personally and actively involved in substantial work leading to the paper, and will take public responsibility for its content.

\section{References}

1. Khandheria BK, Seward JB, Tajik AJ. Transesophageal echocardiography. Mayo Clin Proc. 1994;69:856-63.

2. Michelena HI, Abel MD, Suri RM, et al. Intraoperative echocardiography in valvular heart disease: an evidence-based appraisal. Mayo Clin Proc. 2010;85:646-55.

3. Click RL, Abel MD, Schaff HV. Intraoperative transesophageal echocardiography: 5-year prospective review of impact on surgical management. Mayo Clin Proc. 2000;75:241-7.

4. Nowrangi SK, Connolly HM, Freeman WK, Click RL. Impact of intraoperative transesophageal echocardiography among patients undergoing aortic valve replacement for aortic stenosis. J Am Soc Echocardiogr. 2001;14:863-6.

5. Hoffmann R, Flachskampf FA, Hanrath P. Planimetry of orifice area in aortic stenosis using multiplane transesophageal echocardiography. J Am Coll Cardiol. 1993;22:529-34.

6. Schafers HJ, Schmied W, Marom G, Aicher D. Cusp height in aortic valves. J Thorac Cardiovasc Surg. 2013;146:269-74.

7. Oh CC, Click RL, Orszulak TA, Sinak LJ, Oh JK. Role of intraoperative transesophageal echocardiography in determining aortic annulus diameter in homograft insertion. J Am Soc Echocardiogr. 1998;11:638-42.

8. Currie PJ, Stewart WJ. Intraoperative echocardiography for surgical repair of the aortic valve and left ventricular outflow tract. Echocardiography. 1990;7:273-88.

9. Berrebi A, Monin JL, Lansac E. Systematic echocardiographic assessment of aortic regurgitation - what should the surgeon know for aortic valve repair? Ann Cardiothorac Surg. 2019;8:331-41.

10. Grewal J, Mankad S, Freeman WK, et al. Real-time three-dimensional transesophageal echocardiography in the intraoperative assessment of mitral valve disease. J Am Soc Echocardiogr. 2009;22:34-41.

11. de Kerchove L, Jashari R, Boodhwani M, et al. Surgical anatomy of the aortic root: implication for valve-sparing reimplantation and aortic valve annuloplasty. J Thorac Cardiovasc Surg. 2015;149: 425-33.

12. Karalis DG, Bansal RC, Hauck AJ, et al. Transesophageal echocardiographic recognition of subaortic complications in aortic valve endocarditis. Clinical and surgical implications. Circulation. 1992;86:353-62.

13. Wunderlich NC, Beigel R, Siegel RJ. Management of mitral stenosis using 2D and 3D echo-Doppler imaging. JACC Cardiovasc Imaging. 2013;6:1191-205.

14. Biaggi P, Jedrzkiewicz S, Gruner C, et al. Quantification of mitral valve anatomy by three-dimensional transesophageal echocardiography in mitral valve prolapse predicts surgical anatomy and the complexity of mitral valve repair. J Am Soc Echocardiogr. 2012;25: 758-65.

15. Chotivatanapong T, Lerdsomboon P, Sungkahapong V. Complex surgical repair of rheumatic mitral stenosis. Ann Cardiothorac Surg. 2015;4:480-2.

16. Bakir I, Onan B, Onan IS, Gul M, Uslu N. Is rheumatic mitral valve repair still a feasible alternative?: indications, technique, and results. Tex Heart Inst J. 2013;40:163-9.

17. Schubert SA, Mehaffey JH, Charles EJ, Kron IL. Mitral valve repair: the French correction versus the American correction. Surg Clin North Am. 2017;97:867-88.

18. Ben Zekry S, Nagueh SF, Little SH, et al. Comparative accuracy of two- and three-dimensional transthoracic and transesophageal echocardiography in identifying mitral valve pathology in patients undergoing mitral valve repair: initial observations. J Am Soc Echocardiogr. 2011;24:1079-85.

19. Salem A, Abdelgawad AME, Elshemy A. Early and midterm outcomes of rheumatic mitral valve repair. Heart Surg Forum. 2018;21: E352-8.

20. Adams DH, Rosenhek R, Falk V. Degenerative mitral valve regurgitation: best practice revolution. Eur Heart J. 2010;31:1958-66.

21. Drake DH, Zimmerman KG, Hepner AM, Nichols CD. Echoguided mitral repair. Circ Cardiovasc Imaging. 2014;7:132-41.

22. Chin JH, Lee EH, Choi DK, Choi IC. The effect of depth of anesthesia on the severity of mitral regurgitation as measured by transesophageal echocardiography. J Cardiothorac Vasc Anesth. 2012;26:994-8.

23. Varghese R, Anyanwu AC, Itagaki S, Milla F, Castillo J, Adams DH. Management of systolic anterior motion after mitral valve repair: an algorithm. J Thorac Cardiovasc Surg. 2012;143:S2-7.

24. Marwick TH, Stewart WJ, Lever HM, et al. Benefits of intraoperative echocardiography in the surgical management of hypertrophic cardiomyopathy. J Am Coll Cardiol. 1992;20:1066-72.

25. Sadat K, Diddi HP, Klas B, et al. Live/real time three-dimensional transesophageal echocardiographic assessment of ventricular septal volume and mass before and after myectomy in hypertrophic cardiomyopathy. Echocardiography. 2013;30:1227-31. 
26. Bruce CJ, Nishimura RA, Tajik AJ, Schaff HV, Danielson GK. Fixed left ventricular outflow tract obstruction in presumed hypertrophic obstructive cardiomyopathy: implications for therapy. Ann Thorac Surg. 1999;68:100-4.

27. Kaple RK, Murphy RT, DiPaola LM, et al. Mitral valve abnormalities in hypertrophic cardiomyopathy: echocardiographic features and surgical outcomes. Ann Thorac Surg. 2008;85:1527-35.

28. Kwon DH, Setser RM, Thamilarasan M, et al. Abnormal papillary muscle morphology is independently associated with increased left ventricular outflow tract obstruction in hypertrophic cardiomyopathy. Heart. 2008;94:1295-301.

29. Collis RA, Rahman MS, Watkinson O, Guttmann OP, O'Mahony $\mathrm{C}$, Elliott PM. Outcomes following the surgical management of left ventricular outflow tract obstruction; A systematic review and meta-analysis. Int J Cardiol. 2018;265:62-70.
30. Baumgartner H, Falk V, Bax JJ, et al. 2017 ESC/EACTS Guidelines for the management of valvular heart disease. Eur Heart J. 2017;38: 2739-91.

31. Zoghbi WA, Adams D, Bonow RO, et al. Recommendations for noninvasive evaluation of native valvular regurgitation: a report from the American Society of Echocardiography developed in collaboration with the Society for Cardiovascular Magnetic Resonance. J Am Soc Echocardiogr. 2017;30:303-71.

This material is the authors' own original work, which has not been previously published elsewhere. The paper is not currently being considered for publication elsewhere.

Publisher's note Springer Nature remains neutral with regard to jurisdictional claims in published maps and institutional affiliations. 